112. TREINAMENTO EM CADÁVER DAS TÁTICAS CIRÚRGICAS DE ABORDAGEM DA VEIA CAVA INFERIOR SUPRA-RENAL

Barsella AR, Benavides M, Dall'Olio G, Guimarães SO, Polimanti AC, Velha LPE

samuelguimarães@yahoo.com.br

Introdução: A veia cava inferior (VCI) é a maior veia do corpo e seu estudo anatômico cirúrgico é de suma importância, principalmente no trauma abdominal, pois sabe-se que um em cada 50 ferimentos por arma de fogo e um em cada 300 ferimentos por arma branca lesam a veia cava. A maioria dos óbitos envolvendo lesões de VCI (até 82\%) ocorre nas primeiras 24 horas após a lesão e estão relacionados, principalmente, ao sangramento decorrente das dificuldades próprias do controle das lesões vasculares. A mortalidade das lesões da VCI varia de $21 \%$ a $81 \%$, sendo que no segmento supra-renal é de aproximadamente $70 \%$. Objetivos: Apresentar detalhadamente as manobras para abordagem da VCI em sua porção supra-renal, através de um enfoque anatômico e cirúrgico. Métodos: Foram realizadas dissecações em cadáveres no Serviço de Verificação de Óbito da Universidade de São Paulo, no período de abril de 2001 a junho de 2003, com documentação fotográfica e revisão bibliográfica. Resultados: Elaboração de um pôster mostrando as vias de acesso da VCI entre o fígado e as veias renais, através da manobra de Kocher, que consiste em rebater a segunda e terceira porções do duodeno para a linha média, ressaltando suas intimas relações com órgãos, vasos e estruturas vizinhas. Conclusão: $O$ treinamento em cadáveres nos concede maior precisão, segurança e versatilidade das manobras cirúrgicas, dada a possibilidade de repetição e estudo continuado.

\section{TREINAMENTO EM CADÁVER DE MANOBRAS CRÍTICAS NO MEDIASTINO: ABORDAGEM DO SEGMENTO INTRAPERICÁRDICO DAS ARTÉRIAS PULMONARES}

Barsella AR, Benavides M, Guimarães SO, Penha FG, Polimanti AC, Velha LPE

afonso_sp@yahoo.com.br

Introdução: A abordagem de escolha às lesões vasculares mediastinais é um dos maiores dilemas na cirurgia de trauma. Estudos relatam que 20,1\% dos traumas vasculares situam-se no tórax, e, deste grupo, $71 \%$ dos pacientes com lesões importantes dos vasos da base vão a óbito antes de chegarem ao hospital. No entanto, com a evolução do atendimento préhospitalar, a incidência destas lesões vem aumentando, havendo relatos de pacientes tratados com sucesso. As lesões das artérias pulmonares são encontradas em até $7 \%$ dos pacientes submetidos à toracotomia por trauma penetrante de tórax e a sobrevida destes pacientes é determinada pela rápida abordagem da emergência dos vasos, em seu segmento intrapericárdico. Objetivo: Apresentar detalhadamente as manobras de abordagem as artérias pulmonares, em seu segmento intra-pericárdico. Métodos: Foram realizadas dissecações em cadáveres no Serviço de Verificação de Óbito da Universidade de São Paulo, no período de abril de 2001 a junho de 2003, com documentação fotográfica e revisão bibliográfica. Resultados: Os resultados apresentados nos permitem a elaboração deste pôster com fotos demonstrativas das táticas anátomocirúrgicas das possíveis vias de acesso para a abordagem das artérias pulmonares, em seu segmento intra-pericárdico. Conclusão: O treinamento em cadáveres nos concede maior precisão, segurança e versatilidade das manobras cirúrgicas, dada a possibilidade de repetição e estudo continuado.

\section{TREINAMENTO EM CADÁVER DE MANOBRAS CRÍTICAS EM MEDIASTINO: CLAMPEAMENTO DO HILO PULMONAR}

Barsella AR, Benavides M, Guimarães SO, Polimanti AC, Veiga MG, Velha LPE

afonso_sp@yahoo.com.br

Introdução: Cerca de $15 \%$ dos pacientes vítimas de trauma torácico necessitam de toracotomia de urgência, e $15 \%$ destes, têm a necessidade da realização do clampeamento do hilo pulmonar, por hemorragia severa, destruição parenquimatosa importante, ou ambos. A lesão dos vasos hilares evolui a óbito em até $70 \%$ dos casos. Entre as causas de morte destes pacientes encontram-se a incapacidade de ventilar, sangramento incontrolável, e embolia gasosa. A sobrevida destes pacientes depende da agilidade na hemostasia do hilo. Objetivo: Apresentar detalhadamente as manobras de abordagem das estruturas do hilo pulmonar. Métodos: Foram realizadas dissecações em cadáveres no Serviço de Verificação de Óbito da Universidade de São Paulo, no período de abril de 2001 a junho de 2003, com documentação fotográfica e revisão bibliográfica. Resultados: Os resultados apresentados nos permitem a elaboração deste pôster com fotos demonstrativas das táticas anátomo-cirúrgicas dos acessos ao hilo pulmonar, que consiste na secção do ligamento pulmonar inferior, seguido do rebatimento do pulmão anteromedialmente, permitindo o isolamento e clampeamento das estruturas do hilo. Conclusão: $O$ treinamento em cadáveres nos concede maior precisão, segurança e versatilidade das manobras cirúrgicas, dada a possibilidade de repetição e estudo continuado.

\title{
PÔSTERES CLÍNICOS
}

\section{AVALIAÇÃO DA NEUROPATIA AUTONÔMICA EM PACIENTES DIABÉTICOS}

Barbosa FB, Di Rienzo RT, Fraige Filho F, Gregorio CL, Kusano CT, Santomauro AT

camilatakase@hotmail.com

Introdução: O efeito deletério do diabetes no sistema nervoso autonômico é amplamente conhecido, porém pouco valorizado na prática clínica. Objetivo: Determinar a aplicabilidade e grau de alteração dos testes para avaliação autonômica cardiovascular (variação da freqüência cardíaca durante respiração forçada, durante manobra de Valsalva e com mudança postural; e variação da pressão arterial durante esforço e com mudança de postura) em diabéticos quando comparados a indivíduos normais. Métodos: Foram avaliados 20 diabéticos tipo 2 (grupo I).Todos apresentavam fundo de olho normal, microalbuminúria $<20 \mathrm{mcg} / 24 \mathrm{~h}$, negavam tabagismo e etilismo. Tinham diagnóstico há menos que 15 anos e controle metabólico razoável (hemoglobina glicada $<9,5 \%$ ). O grupo normal (II) negava hipertensão arterial, tabagismo ou etilismo. A comparação dos dois grupos mostrou no "beat to beat" média de 9,7(grupo I) $\mathrm{X} 22,63$ (II) com $\mathrm{p}<0.001$; na manobra de Valsava achou-se $0,85 \mathrm{X}$
1,32 com $\mathrm{p}<0.001$; a relação R-R $30^{\circ} / 15^{\circ}$ foi de $0,86 \times 1,17$ com $\mathrm{p}<$ 0.001 ; o "handgrip" mostrou-se $11, x 24,5 \mathrm{~mm} \mathrm{Hg}$ (p < 0,001); e variação postural com queda da pressão sistólica de 8,2 x 2,0 mm Hg (p < 0.001). O "beat to beat" foi o teste mais alterado ( $89 \%$ dos pacientes) e o menos foi a variação postural da pressão arterial $(40 \%)$. Conclusão: 1 - Os testes autonômicos cardiovasculares são importantes na avaliação dos pacientes diabéticos, apresentando alterações mesmo antes de outras complicações; 2- O "beat to beat" é o teste mais alterado; 3- A realização destes testes deveria ser incluída na avaliação rotineira dos diabéticos pela importância apresentada na prevenção de eventos cardiovasculares.

\section{AVALIAÇÃO DAS CO-MORBIDADES EM CRIANÇAS E ADOLESCENTES OBESOS DE AMBULATÓRIO DE REFERÊNCIA}

Bozzetti RM, Chemiotti P, Fernandes APC, Pitta TS, Sarni RS, Souza FIS robozzetti@uol.com.br

Objetivo: Avaliar a frequência de co-morbidades em crianças e adolescentes obesos e relacioná-las com IMC, cintura abdominal, pressão arterial e risco cardiovascular familiar. Método: Através de estudo retrospectivo e transversal, foram avaliados os prontuários dos pacientes do Ambulatório 
de Obesidade da FMABC. Dados coletados: idade, antecedentes familiares, peso, estatura (OMS,1995), índice de massa corpórea (IMC-CDC,2000), cintura abdominal (CINT,cm-MacCarthy,1998), pressão arterial sistêmica (PAS-sistólica e PAD-diastólica, mmHg Task Force-1996), colesterol tota (CT, mg/dL), frações (VLDL-c, HDL-c, LDL-c - método enzimático, $\mathrm{mg} / \mathrm{dL}$ ), triglicérides (TG-método enzimático, $\mathrm{mg} / \mathrm{dL}$ ) e glicemia (GLICmétodo colorimétrico,mg/dL). Análise estatística: t-student e qui-quadrado. Resultados: Foram estudados 67 pacientes obesos, 31/67(43,3\%) masculino, mediana de idade 9,85 anos, sendo que $27 / 55(49,1 \%$ ) eram pré-púberes. As médias dos z-escore do PE, EI, PI e IMC foram: 3,68; 0,$63 ; 2,91$ e 2,18 ; respectivamente. Antecedentes para doença cardiovascular em parentes de primeiro grau foram referidos em: $29,8 \%$ (obesidade); $7,5 \%$ (dislipidemia); $10,5 \%$ (HAS) e 3\%(diabetes mellitus). Houve inadequação em relação as variáveis estudadas(percentual): cintura de $59 \%$, colesterol total de $32 \%$, LDL de $28 \%$, HDL de $18 \%$, VLDL de $51 \%$, TG de $41 \%$, PAS de $39 \%$, PAD de $36 \%$, glicose de $7,1 \%$. As médias de PAS e PAD estavam associadas as maiores medidas de $\operatorname{CINT}(\mathrm{p}=0,0002$ e $\mathrm{p}=0,034)$. O mesma significância não foi encontrada em relação aos lípides. O IMC associou-se com níveis elevados de $\operatorname{PAD}(\mathrm{p}=0,001)$. Conclusão: Encontramos um grande número co-morbidades na população estudada. Inclusive com repercussões orgânicas, especialmente na pressão arterial, mesmo sendo a maioria prépúberes. Este fato, aliado ao alto percentual de antecedentes familiares positivos, coloca essa população como de alto risco para o desenvolvimento de doença cardiovascular precocemente.

\section{AVALIAÇ̃̃O HISTOMORFOMÉTRICA E ULTRA- ESTRUTURAL DAS PLAQUETAS CIRCULANTES EM GESTANTES NORMOTENSAS E HIPERTENSAS CRÔNICAS: ESTUDO DUPLO- CEGO}

Duarte-Barros ML, Giribela FEG, Simões RS

maristela.dmed@epm.br

Introdução: a hipertensão arterial na gravidez permanece ainda como causa mais freqüente de mortalidade materna e perinatal. Nessa condição clínica ocorrem vasoespasmo generalizado, lesões vasculares, redução plaquetária e alterações na agregação e adesividade plaquetária. Objetivo: avaliar aspectos histológicos e morfométricos das plaquetas circulantes através das microscopias eletrônicas de transmissão e de varredura em gestantes normotensas e hipertensas. Casuística: foram selecionadas sete pacientes grávidas normotensas e oito grávidas hipertensas crônicas atendidas no Hospital São Paulo. Trabalho submetido e aprovado pelo Comitê de Ética da Universidade Federal de São Paulo. Todas as pacientes deram consentimento por escrito. Métodos: após coleta do sangue, as plaquetas foram previamente fixadas e, a seguir, separadas, processadas e incluídas para estudo ao nível de microscopias eletrônicas de transmissão e de varredura. Nas eletromicrografias obtidas foram avaliados parâmetros indicadores de ativação plaquetária. Essa avaliação foi realizada por dois observadores em momentos distintos, sob estratégia duplo-cego, e aplicada análise de concordância. Resultados: houve concordância entre os dois observadores, tendo os dados revelado maior grau de ativação, estatisticamente significante, nas plaquetas de gestantes normotensas quando comparadas às das gestantes hipertensas crônicas. Em contrapartida, encontrouse maior eletrodensidade nas plaquetas das gestantes hipertensas crônicas, porém sem mostrar sinais de ativação quanto à forma, disposição dos grânulos e presença de pseudópodes. Conclusão: as plaquetas de gestantes normotensas apresentaram-se com maior grau de reatividade do que as hipertensas crônicas que têm aspecto desgastado e envelhecido.

\section{CINETOSE: AVALIAÇ̃̃o CLÍNICA E OTONEUROLÓGICA}

Anadão CA, Moio AM, Ogasawara MM, Ramos GM, Rapoport PB, Santos $\mathrm{AF}$

amoio@ig.com.br

Introdução: A cinetose é uma alteração causada pelo movimento. Ela é determinada por um conjunto de sintomas, sendo os principais: náuseas, vômito, sudorese fria e palidez.Um grande número de pessoas já apresentou pelo menos um quadro de cinetose durante a vida, principalmente na idade inferior a dois anos devido à imaturidade da bainha de mielina. A cinetose pode ainda estar relacionada a alterações vestibulares periféricas ou centrais, sensibilidade exacerbada do aparelho vestibular ou do sistema vestíbulovisual, alterações neurológicas ou psicossomáticas e distúrbios das conexões vestibulares visuais e proprioceptivas. Objetivo: Apresentação de dois casos de cinetose com considerações gerais sobre a doença, sua avaliação clínica e otoneurológica. Métodos: No presente estudo, foram selecionados dois pacientes que apresentavam quadro clínico de cinetose. Para avaliação complementar foram realizados anamnese, exame otorrinolaringológico e exame vestibular. Neste último foram analisados: calibração dos movimentos oculares, presença de nistagmo espontâneo, rastreio pendular, nistagmo optocinético, nistagmo per-rotatório e nistagmo per-calórico. Resultados: os pacientes apresentavam crises fortes de vertigem, repentinas e duradouras, causadas pelo movimento. Os exames mostraram-se normais, sendo que uma das pacientes apresentou assimetria na prova rotatória pendular decrescente e decrutamento na prova calórica. Conclusão: Verifica-se que esta patologia gera um grande desconforto, restringindo as atividades diárias e prejudicando a qualidade de vida dos pacientes. Apesar da normalidade dos exames, o quadro clínico dos pacientes permitiu a confirmação do diagnóstico de acordo com o que é descrito na literatura

\section{DOENÇAS MUCOCILIARES: RELATO DE CASO E REVISÃO DA LITERATURA}

Mendonça RR, Moretti G, Neves LAC, Queiroz GAS, Rapoport PB, Zanini $\mathrm{R}$

gmorettijr@uol.com.br

Objetivo: O clearence mucociliar é uma parte importante da defesa inespecífica das vias aéreas. O batimento coordenado dos cílios do nariz, traquéias e brônquios possibilita a mobilização da camada de muco e com ele microorganismos inalados e outro partículas em direção à faringe. Este trabalho tem como objetivo apresentar as principais doenças mucociliares, destacando sinais e sintomas apresentados pelos pacientes pediátricos e orientando sobre exames complementares para definir a etiologia. Entre as doenças estudadas daremos ênfase à Discinesia Ciliar Primária, Síndrome de Young e Fibrose Cística. Discussão: L.A.S., masculino, procurou o serviço de Otorrinolaringologia do $\mathrm{ABC}$ por obstrução nasal há 4 anos, pior à direita, associada a rinorréia posterior e tosse, com melhora após uso de antibiótico. Em tratamento prévio em outro serviço houve indicação cirúrgica por sinusite crônica, porém, a cirurgia foi suspensa por alteração pulmonar. Em investigação pneumológica, foram detectadas bronquiectasia em base direita, atelectasia e condensação em pulmão direito. Atualmente os sintomas regrediram com uso de antibiótico por vinte e um dias, uso diário de fluidificante e lavagem nasal. Realiza em nosso serviço investigação de doença sistêmica pela associação de sinusite crônica e bronquiectasia incomum. Conclusão: Durante a investigação de sinusites crônicas em pacientes pediátricos, devemos ter em mente os principais diagnósticos diferenciais, incluindo as doenças sistêmicas discutidas nesse trabalho para oferecer ao paciente o diagnóstico preciso de sua doença e orientação terapêutica mais adequada.

\section{EQUOTERAPIA: UMA NOVA MODALIDADE TERAPÊUTICA PARA OS PROFISSIONAIS DA SAÚDE}

\section{Campos TY, Castello GL, Lara SRG}

thaisyamasaki@ig.com.br

Introdução: Entre os programas de reabilitação convencionais e novos, encontra-se a equoterapia que caracteriza-se pela utilização do cavalo para fins terapêuticos dentro de uma abordagem interdisciplinar, na qua deve ser realizado por profissionais legalmente habilitados. Em 1997, a equoterapia foi reconhecida pelo Conselho Federal de Medicina, como método terapêutico de reabilitação de pessoas portadoras de deficiência físicas, mentais e/ou necessidades especiais, buscando o desenvolvimento bio-psico-social dos mesmos, considerando ainda, que sua aplicação fica restrita a equipe interdisplinar e sempre com indicação médica. Objetivo: Apresentar aos profissionais da saúde uma nova modalidade terapêutica Métodos: busca de artigos sobre o assunto na ANDE-BRASIL (Associação 
Nacional de Equoterapia), selecionando os artigos mais pertintentes. Conclusão: este tipo de terapia, por estar centrada na individualidade de cada praticante, permite que os objetivos de diferentes áreas possam ser alcançados ao mesmo tempo, ou seja, é um método terapêutico onde profissionais como o médico, o enfermeiro, o fonoaudiólogo, o fisioterapeuta, o psicólogo entre outros podem atingir objetivos gerais e específicos.

\section{ESCLEROSE TUBEROSA - RELATO DE CASO}

Bertolami A, Chaves FP, Hanaina M, Moraes KCFV, Mozeti V, Possendoro KA kcfvm@hotmail.com

Introdução: A Esclerose Tuberosa (ET) ou Doença de Bourneville é uma doença hereditária, neurocutânea e multisistêmica que se caracteriza pela presença de hamartomas em múltiplos órgãos como sistema nervoso central, rins, pele, coração, pulmão, olhos e ossos. A ET tem herança autossômica dominante, associada a uma mutação nos cromossomos 9q34 (ET tipo 1) e 16p13 (ET tipo 2), sendo o tipo 2 mais comum. Suas manifestações clínicas em sua forma clássica se caracterizam por epilepsia, retardo mental e angiofibromas faciais, caracterizando a tríade de Vogt. Segundo alguns autores, a ET tem incidência de 1:10.000 e não há preponderância racial ou de sexo. Discussão do Caso: Relata-se um caso de paciente de 33 anos com esclerose tuberosa diagnosticada com a utilização de dados clínicos e exames de imagem, segundo os preceitos atuais. Deu-se ênfase para o diagnóstico não invasivo, uma vez que a paciente não apresentava condições clínicas para ser submetida a procedimentos diagnósticos invasivos.

\section{ESTEATOSE HEPÁTICA EM CRIANCAS DE ESCOLA PÚBLICA DO BAIRRO DE CAPUAVA - MUNICÍPIO DE SANTO ANDRÉ - SÃO PAULO}

Chemiotti P, Fernandes APC, Pitta TS, Rocha KG, Sarni RS, Souza FIS.

\section{rssarni@uol.com.br}

Objetivo: Avaliar a freqüência de esteatose hepática (EH) por ultrassonografia (USG) e relacioná-la com provas de função hepática (PFH), condição nutricional, perfil lipídico (triglicérides-TG,colesterol total-CT e fraçõesLDL-c,VLDL-c,HDL-c), peroxidação lipídica (TBARS) e níveis séricos de retinol (RET) e carotenóides (CAROT) em escolares. Métodos: Em estudo transversal/controlado foram avaliadas 91 crianças pré-púberes (46-eutróficas e 45-obesas), mediana de idade 8,6anos, baixo nível sócioeconômico, de escola pública de Capuava/Santo André-SP. Foram coletados os seguintes dados: peso, estatura (OMS,1995), cintura (MacCarthy,1999), PFH-TGO/TGP/Gama-GT (método colorimétrico), USG-hepática, TBARS (espectrofotometria), RET e CAROT (Bessey-Lowry), CT e frações (método enzimático) e TG (método enzimático). Estatística: Qui-quadrado e Teste de Fisher. Resultados: Encontramos EH difusa em 48/91(52,74\%) dos escolares, sendo: 40/48(83,3\%) discreta, 7/48(14,5\%) moderada e $1 /$ $48(2,1 \%)$ grave. Verificamos que $5 / 7(71,4 \%)$ crianças com EH- moderada eram obesas. Observamos deficiência em 28 e $100 \%$ das crianças para RET e CAROT, respectivamente. Não se verificou relação significativa entre PFH alteradas e EH com a condição nutricional. As crianças PFH alteradas (17/91-19\%) apresentaram elevação significativa do LDL$\mathrm{c}(\mathrm{p}=0,023)$ e redução significativa do $\operatorname{HDL}-\mathrm{c}(\mathrm{p}=0,0008)$ em relação às com PFH normais. EH não mostrou associação significativa com cintura, PFH, perfil lipídico, TBARS e níveis séricos de RET e CAROT. Conclusão: Detectou-se alta frequiência de EH na população estudada, o que pode ser decorrente do comprometimento da defesa antioxidante, evidenciada neste estudo pela deficiência de CAROT. A ausência de relação com a condição nutricional também é vista em outros estudos da literatura. As PFE mostraram-se mais preditivas de dislipidemias do que a EH avaliada pela ultrassografia.

\section{HIPOTENSÃO ORTOSTÁTICA EM IDOSOS AMBULATORIAIS}

Grizante P, Lima NCP, Lopes LS, Murrer G, Valente M

leo.s1@ig.com.br
Introdução: A hipotensão ortostática (HO) é definida como uma queda na pressão arterial sistólica (PAS) de $20 \mathrm{mmHg}$ ou mais e/ou uma queda na pressão arterial diastólica (PAD) de $10 \mathrm{mmHg}$ ou mais quando se assume a posição ortostática. Pode estar associada ou não a sintomas, e a HO tem sido associada a quedas nos idosos. Objetivo: avaliar a prevalência de HO e seus sintomas nos pacientes ambulatoriais e identificar seus principais fatores de risco. Métodos: 50 idosos com idade igual ou superior a 60 anos (média 71,48) de ambos os sexos (18 homens e 32 mulheres) que procuraram o ambulatório da FMABC participaram deste estudo. A pressão arterial (PA) foi medida na posição supina, após repouso de 5 minutos nesta mesma posição. Foi solicitado ao paciente que ficasse na posição ortostática, e então aferida sua PA em 1 e 3 minutos após ter assumido esta posição. O paciente foi questionado sobre os sintomas e fatores de risco de HO. Resultados: A prevalência de $\mathrm{HO}$ foi de $38 \%$ sendo que destes apenas $11 \%$ apresentou sintomas. Alteração em PAS e PAD concomitante foi mais frequiente (12 casos). Um ou mais fatores de risco foram encontrados em todos os pacientes sendo o uso de medicamentos e a hipertensão os mais freqüentes. Conclusão: Existe alta prevalência de HO em idosos ambulatoriais, o que deve atentar ao médico a aferição da PA corretamente, principalmente em idosos com uso de medicamentos e hipertensos.

\section{HISTOPLASMOSE COMO CAUSA DE INSUFICIÊNCIA ADRENAL PRIMÁRIA}

Barbosa FB, Di Rienzo RT, Fraige Filho F, Gregorio CL, Kusano CT, Santomauro AT

febsbarbosa@hotmail.com

Introdução: $\mathrm{O}$ achado incidental de uma massa em região da supra-renal é uma ocorrência cada vez mais freqüente na prática médica, necessitando de avaliação clínica cuidadosa para verificar outras causas de insuficiência adrenal e evitar indicação cirúrgica desnecessária. Discussão do Caso: Descreve-se um paciente do sexo masculino, de 55 anos, com quadro de perda de 6 quilos nos últimos seis meses e que ao ultrassom de abdômen mostrou imagem sugestiva de massa em região de supra-renal direita com $4,2 \times 2,3 \mathrm{~cm}$, sendo indicado cirurgia para retirada do tumor. Na avaliação pré-operatória, o paciente vinha evoluindo com astenia e fraqueza muscular progressivas e episódios freqüentes de náusea e vômitos, associado a diarréia .Ao exame físico apresentava PA 120 × $80 \mathrm{mmHg}$ deitado e 90 × 60 $\mathrm{mmHg}$ em pé, escurecimento gengival e cicatricial. Os exames laboratoriais mostravam $\mathrm{Na}=142 \mathrm{mEq} / \mathrm{L}, \mathrm{K}=5,0 \mathrm{mEq} / \mathrm{L}$, leucopenia $\left(3000 / \mathrm{mm}^{3}\right)$ com linfocitose $(54 \%)$ e eosinofilia $(10 \%)$. A tomografia de abdômen mostrou aumento bilateral das supra-renais, sendo indicado biópsia percutânea da supra-renal direita, orientada pela tomografia e o estudo histopatológico mostrou presença de Histoplasma capsulatum. Descartouse, então a indicação de cirurgia, sendo instituído tratamento com Anfotericina B e cetoconazol, além da reposição hormonal adrenal. Conclusão: Conclui-se que o achado incidental de imagens em região de supra-renal deve ser acompanhado de uma investigação clínica cuidadosa, para evitar-se indicar uma cirurgia que muitas vezes pode se mostrar desnecessária.

\section{HOMOCISTEÍNA: O COLESTEROL DO SÉCULO XXI}

Diniz RW, Grigio JV, Napoli FC, Pontes MF, Saggiomo JVA, Sampaio KO renatawdiniz@yahoo.com.br

Introdução: Evidências acumuladas desde os trabalhos de McCully apontam a homocisteína como fator de risco importante e independente para síndromes coronárias e demais processos ateroscleróticos. Existem mecanismos propostos para o envolvimento da homocisteína na lesão endotelial. A hiperhomocisteinemia produz danos às células endoteliais principalmente por oxidação, produzindo radicais livres, hiperóxidos de hidrogênio, este particularmente implicado como causa principal de dano. Métodos: Este trabalho é uma revisão bibliográfica de estudos, que relacionam a homocisteína ao infarto agudo do miocárdio datados de 1969 a 2001. Desenvolvimento: Sabe-se que a homocisteína combinase com a lipoproteína de baixa densidade (LDL), fornecendo outra fonte 
de oxigênio reativo; esta espécie de oxigênio contribui para a deficiência orgânica do endotélio, também aumentam a concentração de oxicolesteróis aterogênicos, a peroxidação de lipídeos e oxidação de LDL, finalmente a hiperhomocisteinemia predispõe o acúmulo de plaquetas. Essa entidade é causada por uma deficiência enzimática ou de seus cofatores (ácido fólico - B9, piridoxina - B6 e cianocobalamina - B12). As enzimas envolvidas são: cistationina-B-sintase, N5- N10-metiltetrafolato-redutase e metilcobalamina sintase. Conclusão: Ensaios clínicos quanto ao tratamento da homocisteína não são conclusivos, entretanto, quando se propõe o tratamento, seria com B6, B9, B12, pois agem aumentando a metabolização da homocisteína à metionina. Estudos estão sendo realizados para determinarmos o real papel da homocisteína e do seu tratamento no processo da ateromatose.

\section{IMPLANTAÇÃO DO AMBULATÓRIO VASCULAR PARA CLAUDICAÇÃO INTERMITENTE: METODOLOGIA PARA AVALIAÇÃO DO CLAUDICANTE}

Catelane LGC, Debs PGK, Hirai LK, Kafejian O, Oliveira FP, Yamasaki RY

fpmarini@uol.com.br

Introdução: A claudicação intermitente(CI), sintoma patognomônico da doença arterial aguda oclusiva periférica (DAAOP), restringe as atividades profissionais e sociais do claudicante. Em cerca de $17 \%$ dos pacientes, a DAAOP evolui para isquemia grave de membros inferiores (MMII) e 3\% a 7\% apresentam risco de mortalidade maior que o risco de perda do membro. Assim, o diagnóstico e o acompanhamento ambulatorial desses pacientes tornam-se necessários na prevenção e na evolução das DAAOP. A avaliação de pacientes sintomáticos para CI prevê a instalação de esteira eletrônica, aparelho de ultra-sonografia com Doppler portátil, esfignomanômetros adequados para a medição de níveis pressóricos segmentares(NPS) dos MMII. Métodos: Estudo da evolução da DAAOP em 83 pacientes do ambulatório de vascular da FMABC, no período entre março de 2002 a maio de 2003, a partir de um protocolo baseado na história clínica, exame físico e fatores de risco. Os pacientes foram submetidos à medição da NPS nos MMII juntamente com testes em esteira. Exames laboratoriais, avaliação cardiológica e duplex scan de aorta, artérias ilíacas e artérias de MMII também foram solicitadas. Resultados: De 83 pacientes com faixa etária entre 34 a 82 anos, de ambos os sexos, 58 claudicantes manifestaram dor unilateral, com presença de compormetimentos fêmoro-poplíteo (56,9\%), ilíaco-femoral $(24,1 \%)$, arterial aortoilíaco(12\%) e segmento poplíeo abaixo(7\%). Conclusão: A implantação do laboratório vascular para avaliação inicial, abordagem clínica atenciosa e orientação adequada dos pacientes promove a conscientização e melhor adesão ao tratamento a esta categoria de pacientes.

\section{ISQUEMIA MIOCÁRDICA INDUZIDA PELA INFUSÃO DE 5- FLUOROURACIL}

Bordinhon TS, Ferlin F, Ferreira C, Gomes ALR, Meneghini A, Santos RK adrianagomes_32@hotmail.com

Introdução: O 5-FU é um antimetabólito usado no tratamento quimioterápico em diversas neoplasias. Diversos autores reportam uma possível cardiotoxicidade relacionada com a droga, cuja incidência varia entre 1,2 a 18\%. Relato de Caso: Paciente masculino, 50 anos, portador de AIDS, carcinoma espino celular de canal anal e uso crônico de drogas antiretrovirais, sem antecedentes cardiológicos. Após infusão de 5-Fluorouracil (5-FU), apresentou dor precordial típica e prolongada, que cedeu com Nitroglicerina (NTG) endovenosa. Foi submetido a estudo eletrocardiográfico (ECG), ecocardiograma, dosagem de enzimas cardíacas e cinecoronariografia. As dosagens de enzimas cardíacas e o ecocardiograma foram normais. O ECG de repouso mostrou isquemia subendocárdica em parede ântero-septal do ventrículo esquerdo que se normalizou após o uso de NTG. Realizada Cinecoronariografia que mostrou artérias coronárias e ventrículo esquerdo normais. Comentários: O mecanismo da cardiotoxicidade do 5-FU ainda não foi completamente elucidado, podendo ser: 1. reação auto-imune; 2. reação inflamatória; 3. efeito tóxico direto sobre as células miocárdicas; 4. pericardite; 5. espasmo coronariano 2, 3 e 4 Atualmente, a teoria mais aceita é a de espasmo coronário. O levantamento da literatura revela que a complicação é bastante rara, aventando-se a participação do endotélio alterado pelo uso crônico de anti-retrovirais, e de espasmo coronário desencadeado pelo citostático. As alterações metabólicas causadas pelo uso de inibidores de proteases têm como efeito colateral principal a ocorrência de doença arterial coronária prematura, o que, provavelmente, foi o fator desencadeante neste caso. Embora rara, a complicação deve ser lembrada e a infusão de 5-fluorouracil interrompida, especialmente nos pacientes submetidos previamente a tratamento com drogas anti-retrovirais.

\section{MANIFESTAÇÕES DERMATOLÓGICAS INUSITADAS ICONOGRÁFICAS DA HANSENÍASE}

\section{Alessi R, Canavezzi AZ, Cvintal V, Ito LM, Novais MAB, Xavier WC \\ alessi@uol.com.br}

Introdução: A hanseníase, doença de crescente incidência em países como o Brasil, devido à exuberância do quadro clínico, manifesta inúmeras alterações e diversos sinais cutâneos. Muitas vezes, tais manifestações são características e, por meio de profissionais preparados, poderiam ser diagnosticadas precocemente, evitando resultados incapacitantes e muitas vezes irreversíveis. Métodos: Por meio de uma seleção de 52 pacientes hansênicos do ambulatório de Hanseníase do Centro de Saúde Escola Capuava, ligado à Disciplina de Dermatologia da Faculdade de Medicina da Fundação ABC, seis foram selecionados devido a manifestações inusitadas. Resultados: Abordou-se os valores iconográficos nos seis pacientes selecionados segundo quatro manifestações: (I) sinal cubitelar, cicatriz atrófica, acrômica e anestésica de localização bilateral - típica da hanseníase virshowiana; (II) sinal da orelha em brinco, lóbulos auriculares visivelmente pendurados devido a hipertrofia local e causados por infiltrados virshowianos, geralmente acompanhados por facies leonina; (III) sinal do botão, neurite (principalmente do nervo ulnar) que impossibilita movimentação motora das mãos gerando ato dificultoso e movimentos lentos com mão em garra - geralmente nas formas dimorfas da doença; (IV) cianose palmar em topografia de inervação ulnar, a cianose intensa pode proceder a alteração sensitiva e motora do nervo ulnar - manifestação das formas tuberculóides. Conclusão: apesar dos sinais apresentados acima serem pouco descritos em literaturas didáticas, o conhecimento da existência dos mesmos poderia auxiliar no diagnóstico precoce da hanseníase impedindo a evolução da doença para formas clínicas de pior prognóstico.

\section{PARALISIA PERIÓdICA E TIROTOXICOSE}

Barbosa FB, Di Rienzo RT, Ducatti LSS, Fraige Filho F, Kusano CT, Santoumauro AT

rienzo@uol.com.br

Introdução: As manifestações neuromusculares estão presentes em 60 a $80 \%$ dos portadores de tireotoxicose. Entre elas, destaca-se a paralisia periódica da tireotoxicose (PPT), caracterizada por episódios de fraqueza muscular. Discussão do Caso: Descreve-se o caso de um paciente do sexo masculino com 42 anos, que referia há três meses, fraqueza muscular em membros inferiores que durava aproximadamente 2 dias e ocorria a cada 15 dias. Ao exame físico apresentava $\mathrm{PA}=120 \times 80 \mathrm{~mm} \mathrm{Hg}$, pulso $=98$ bpm, exoftalmo bilateral, Sinal de Moebius presente, "lid-lag" presente bilateralmente. Tireóide aumentada de volume duas vezes, fibro-elástica, móvel, indolor. Tremor de extremidades presente. Avaliação neurológica mostrava diminuição de força muscular em membros inferiores, com sensibilidade preservada. Exames Laboratoriais: $\mathrm{Na}=140 \mathrm{mEq} / \mathrm{l}, \mathrm{K}=3,9$ $\mathrm{mEq} / \mathrm{l}, \mathrm{Glicemia}=80 \mathrm{mg} / \mathrm{dl}, \mathrm{Ca}=9,0 \mathrm{mg} / \mathrm{dl}, \mathrm{P}=4,2 \mathrm{mg} / \mathrm{dl}, \mathrm{T} 3=239 \mathrm{ng} / \mathrm{dl}$, $\mathrm{T} 4=19 \mathrm{mcg} / \mathrm{dl}, \quad \mathrm{T} 4$ livre $=6,1 \mathrm{mg} / \mathrm{dl}(0.5-1.2) ; \mathrm{TSH}=0,01 \mathrm{mU} / \mathrm{ml}$, Anticorpo Antitireoglobulina +, Anticorpo AntiTPO +, TRAb +, Liquor normal. Mapeamento e Captação de Tireóide - 42\% em $2 \mathrm{~h}$ e $76 \%$ nas $24 \mathrm{~h}$, mostrando aumento difuso da glândula. Paciente recebeu prednisona $60 \mathrm{mg} / \mathrm{dia}$ (5 dias), PTU $600 \mathrm{mg} / \mathrm{dia}$ e propranolol $120 \mathrm{mg} / \mathrm{dia}$, e encontrase há 4 meses sem sintomatologia. Conclusão: A paralisia periódica da tireotoxicose é muito semelhante à paralisia periódica hipocalemica 
primária (PPHP), onde encontra-se níveis baixos de potássio durante os ataques, sendo importante o diagnóstico diferencial entre estas duas situações, pois diferentemente da PPHP a PPT é curada com a normalização da função tireoidiana.

\section{PLASMOCITOMA INTESTINAL EM PACIENTE COM GAMOPATIA MONOCLONAL}

Chaves FP, Garrido TF, Mader AMAA, Moraes KCFV, Possendoro KA Silva KA

kcfvm@hotmail.com

Introdução: Plasmocitoma extra-ósseo é um raro tipo de neoplasia, sendo mais freqüente na cavidade oral ou trato respiratório superior. Dados Clínicos e Demográficos: Paciente masculino, de 33 anos, branco, procurou o pronto-socorro com queixa de dor e distensão abdominal, perda de peso e melena há 1 mês. Métodos Diagnósticos: O RX de abdome mostrou oclusão intestinal baixa. Foi submetido à laparotomia, com ressecção de íleo terminal e cólon ascendente. Achados Macroscópicos: Havia tumor sobrelevado em ceco, medindo $4,5 \mathrm{~cm}$, com padrão de crescimento infiltrativo ao corte. Achados Microscópicos: Neoplasia composta por células atípicas, com diferenciação plasmocitóide e monoclonalidade para cadeia lambda ao exame imunohistoquímico. Em vista destes achados, foi detectado aumento de dosagem sérica de IgG (4,276 g/ 1) e presença de paraproteínas na região da eletroforese. Não haviam lesões osteolíticas e proteína de Bence Jones na urina; a biópsia de medulla óssea revelou plasmocitose menor do que $10 \%$. Por ter evoluído com recidiva neoplásica em intestino delgado, foi introduzida quimioterapia, com melhora do quadro clínico. Comentário: Os autores acreditam que o comprometimento gastrointestinal de caráter recidivante, como no presente caso, mesmo sem os demais critérios diagnósticos, seja um estadio prévio de evolução ao Mieloma Múltiplo.

\section{RADIOCIRURGIA ESTEREOTAXICA NO TRATAMENTO DOS TUMORES HIPOFISÁRIOS}

Di Rienzo RT, Fraige Filho F, Gregorio CL, Kusano CT, Minin PHP, Santomauro AT

carlinhagregorio@ig.com.br

Introdução: Os tumores pituitários representam 10 a $12 \%$ dos tumores intracranianos. O tratamento pode ser com cirurgia, e/ou radioterapia, e/ ou uso de medicamentos, porém nenhuma modalidade de tratamento tem resultados plenamente satisfatórios. Embora muitos pacientes com microadenomas possam ser curados pela cirurgia transesfenoidal, os resultados são menos satisfatórios com os macroadenomas, principalmente aqueles com extensão supra e/ou paraselar, sendo então necessária complementação com radioterapia. Discussão do Caso: Neste trabalho relata-se a evolução de dois pacientes portadores de tumor hipofisário produtor de $\mathrm{GH}$, que após a cirurgia mantiveram níveis elevados de $\mathrm{GH}$ e somatomedina (IGF-I), sendo então submetidos a radiocirurgia estereotáxica, que diferentemente da radioterapia convencional está relacionada com uma menor ocorrência de panhipopituitarismo e uma maior porcentagem de cura. No caso 1, o paciente apresentou normalização dos níveis de $\mathrm{GH}(<1 \mathrm{mg} / \mathrm{ml}$ durante curva glicêmica) em 4 meses e normalização dos níveis de IGF-I em 8 meses. No caso 2, o paciente normalizou os níveis de GH em 18 meses e de IGF-I em 3 anos. Os dois pacientes preservaram o restante da função hipofisária normal. Conclusão: Conclui-se que a radiocirurgia estereotáxica é uma forma eficaz para tratamento dos tumores hipofisários como complemento a cirurgia e tem menor chance de ocorrência de panhipopituitarismo quando comparado com a radioterapia convencional.

\section{RECÉM NASCIDO PREMATURO PORTADOR DE SÍNDROME DE WILLIANS-BEUREN ACOMPANHADO NO MÉTODO CANGURU: RELATO DE CASO}

Aragão MC, Chamelian MD, Furlan M, Generoso RAM, Silva IS, Zanini RVR

mifurlan@terra.com.br
Objetivo: Os autores relatam caso de prematuro portador de Síndrome de Williams-Beuren (SWB) acompanhado no Método Canguru (MC). O relato se dá pela raridade da síndrome, prematuridade associada e ausência na literatura de tal acompanhamento. Relato de Caso: Recém-nascido (19/ 03/03) de parto normal, Apgar 6/8, Capurro $33^{1 / 7}$ semanas, $1905 \mathrm{~g}$, apresentando sopro cardíaco. Ecocardiograma com resultado CIV de $3 \mathrm{~mm}$, estenose pulmonar pós valvar, PCA de $4 \mathrm{~mm}$ e aumento das cavidades direitas. Apresentava-se hipoativo, fácies típica, estrábico, palato em ogiva, baixa implantação de orelhas com pavilhões mal formados, fronte e fontanelas amplas, sendo eleito para MC. Recebeu alta (28/04/03) com diagnóstico de recém-nascido pré-termo, adequado para idade gestacional, cardiopatia congênita e SWB a esclarecer - cariótipo em análise. Discussão: A SWB é uma desordem autossômica dominante resultante da haploinsuficiência de genes localizados na banda cromossômica 7q11.23, envolvendo o sistema cardiovascular, nervoso central e tecido conectivo. Os aspectos faciais se assemelham a um "delfo", ocorrendo alterações nasais, bucais, oculares, dermatológicas e inúmeras anomalias congênitas. O diagnóstico é clínico, confirmado com a hibridização in situ por fluorescência. Os sentimentos de culpa e rejeição, freqüente nas famílias de recém-nascidos sindrômicos, tornam sua estimulação insuficiente, fator agravante do retardo cognitivo. Assim a Equipe Canguru elegeu essa família para Método Mãe Canguru, pelo melhor atendimento aos prematuros, para estimular o vínculo familiar minimizando a situação supracitada. Conclusão: A construção do vinculo familiar superou os sentimentos conflitantes iniciais de culpa e rejeição, fundamental para melhor inserção social desse recém-nascido, bem como para a saúde familiar, em seu conceito mais amplo.

\section{SÍNDROME CERVICAL - RELATO DE CASO}

Anadão AC, Barroso JC, Corpa JHN, Horie AS, Rapoport PB, Takashima KK

jesse.corpa@terra.com.br

Objetivo: disponibilizar à consulta medica um relato de caso de uma síndrome vertiginosa que se encaixou no quadro de síndrome cervical, desencadeada por uma afecção óssea cervical, assim como seu diagnostico e tratamento. Discussão: A síndrome cervical caracteriza-se por crises vertiginosas recorrentes associadas a queixas cervicais. Trata-se de um tema ainda repleto de controvérsias em relação a sua definição, incidência e sintomatologia. Aceitam-se três mecanismos para a explicação de sua gênese a partir da região cervical: insuficiência vértebro-basilar, disfunção proprioceptiva e estimulação do simpático cervical posterior. A clínica varia de acordo com a etiologia, sendo os sintomas mais freqüentes: tonturas, zumbido, formigamento na hemi-face e membro superior homo-lateral, estalos na região cervical, cefaléia e dor na região cervical. Descrevemos o caso de uma paciente com quadro de vertigem subjetiva e dor cervical posterior que, depois de descartadas as outras hipóteses, foi diagnosticada síndrome cervical. Recebeu vários tratamentos medicamentosos, mas obteve mais sucesso com a fisioterapia. Conclusão: Trata-se de doença de importante diagnóstico diferencial com outras síndromes vertiginosas, pois compromete de maneira significativa a qualidade de vida do paciente.

\section{SÍNDROME DE BERNARD-SOULIER: RELATO DE CASO}

De Moricz R, Moio AM, Ogasawara MM, Ramos GM, Rapoport PB, Santos AF

\section{andrea@danidrea.com.br}

Objetivo: relatar um caso da síndrome de Bernard-Soulier, tendo em vista sua raridade e importância no diagnóstico diferencial das síndromes de epistaxes recorrentes. Discussão de Caso: a síndrome de BernardSoulier é um distúrbio de coagulação congênito raro, causado por anormalidades no receptor do fator de von Willebrand da membrana plaquetária. Clinicamente, exterioriza-se por sangramento cutâneomucoso intenso com início na infância e laboratorialmente, há macrotrombocitopenia. Rela-tamos o caso de LM, sexo feminino, 7 anos, com queixa de epistaxes de repetição desde a primeira infância, hematomas cutâneos disseminados pelo corpo e já apresentou também sangramento digestivo. Nos episódios 
mais intensos de epistaxe, é necessária a transfusão de plaquetas por plasmaferese. Conclusão: a Síndrome de Bernard-Soulier, apesar de rara, deve fazer parte da investigação diagnóstica nos casos epistaxes recorrentes, principalmente quando intensas e com início na infância.

\section{SÍNDROME DE VAN DER HOEVE E KLEYN: RELATO DE CASO}

Appolonio F, Aragão MC, Furlan M, Rapoport PB, Yoshimura R, Zanini RVR

raulzani@ajato.com.br

Objetivo: A proposta dos autores é relatar o caso de um paciente portador da síndrome de Van der Hoeve e Kleyn, seguido de um breve levantamento bibliográfico sobre esta rara doença. Relato de Caso: M.C.N.A., 60 anos, sexo feminino, branca, com queixa de zumbido constante na orelha esquerda, tipo "chiado", iniciado há 10 anos, acompanhado de hipoacusia progressiva ipsilateral. Ao exame físico chamava atenção o aspecto azulado de suas escleras. Interrogada sobre antecedentes mórbidos a paciente referiu múltiplas fraturas desde a adolescência. A otoscopia estava normal. A audiometria tonal demonstrou disacusia condutiva e curva timpanométrica $A r$ em orelha direita. A hipótese diagnóstica foi osteogênese imperfeita com acometimento otológico: síndrome de Van der Hoeve e Kleyn. Discussão: Em 1918, Van der Hoeve e de Kleyn descreveram o primeiro caso de um paciente que sofria de fraturas espontâneas, possuía esclera azul e perda auditiva. Desde então, tem se dado mais atenção à osteogênese imperfeita, uma doença de transmissão autossômica dominante, caracterizada por um defeito na síntese de colágeno tipo I, que impede a ossificação normal da cortical do osso associado a hipoacusia. A audição é afetada em 20 a $40 \%$ dos portadores, sendo clínica e radiologicamente semelhante a otospongiose, uma patologia também caracterizada por displasia óssea. A estapedotomia apresenta bons resultados na reabilitação audiológica desses pacientes. Conclusão: A otospongiose e a síndrome de Van der Hoeve e Kleyn apresentam características otológicas, clínicas e radiológicas muito semelhantes, sendo necessários mais estudos para esclarecer as relações entre ambas patologias.

\section{UTILIZAÇÃO DA DIETA CETOGÊNICA NO TRATAMENTO DE EPILEPSIA REFRATÁRIA: UMA REVISÃO DA LITERATURA}

\author{
Alessi R, Cvintal v, Felgueira RM, Wajjnsztejn R
}

alessi@uol.com.br

Introdução: A Dieta Cetogênica (DC) é uma dieta com alto teor de gordura, baixo teor de proteína e carboidratos usada no tratamento de epilepsia refratária em crianças, desde a década de 20. Objetivo: Revisar sistematicamente e sintetizar as evidências de resposta à DC na redução das crises epilépticas em crianças com epilepsia refratária, assim como os efeitos colaterais apresentados. Métodos: Trata-se de estudo de revisão da literatura, realizado através de busca na Medline, utilizando-se como palavras-chaves: Dieta Cetogênica e epilepsia refratária. Selecionamos para revisão ensaios clínicos que avaliaram a resposta à dieta e seus efeitos colaterais. Resultados: Os critérios de efetividade foram: 1) completa eliminação das crises epilépticas; 2) >90\% de melhora das crises e 3) Entre $50-90 \%$ de redução das crises. Os principais efeitos colaterais relatados durante o período do jejum foram: desidratação, hipoglicemia, vômitos, diarréia e recusa alimentar. Após este período, as complicações mais freqüentemente observadas foram: nefrolitíase, infecções de repetição, hiperuricemia, hipercalcemia, acidose, hipercolesterolemia, irritabilidade, letargia, neuropatia óptica, recusa alimentar e distúrbios gastrointestinais, como náuseas, vômitos, constipação, diarréia e dor abdominal. Conclusão: As série revisadas suportam que cerca de $50 \%$ das crianças submetidas à DC para tratamento de epilepsia refratária apresentam uma melhora significativa $(>50 \%)$ no controle das crises epilépticas, efetividade esta maior que a de muitas drogas anti-epilépticas de nova geração, com um custo muito menor. Os efeitos colaterais apresentados não foram relevantes a ponto de limitar a aplicação da dieta.

\section{PÔSTERES EPIDEMIOLÓGICOS}

\section{A EQUOTERAPIA NA REABILITAÇ̃̃o DE CRIANÇA COM NECESSIDADES HUMANAS BÁSICAS AFETADAS}

Campos TY, Castello GL, Lara SRG

thaisyamasaki@ig.com.br

Introdução: Um terço das doenças infantis acomete o sistema nervoso ou muscular. Muitas doenças são limitadas, predominam ou tem seu início na infância, como a epilepsia, as encefalopatias de origem pré ou perinatal, as afecções musculares, as doenças metabólicas hereditárias, os distúrbios congnitvos e comportamentais entre outros. A identificação, acompanhamento e tratamento de fatores debilitantes da realização de qualquer atividade, devem ser realizados através de programas de reabilitação. Objetivos: Identificar as doenças mais freqüentes entre a população infantil estudada; identificar as melhorias encontradas em crianças com idade de 0 a 10 anos apresentando necessidades humanas básicas afetadas que praticam equoterapia por no mínimo seis meses. Métodos: Foi realizado estudo quantitativo e de levantamento através de questionário aplicado em 14 crianças de 0 a 10 anos que apresentavam necessidades humanas básicas afetadas, que praticam equoterapia por no mínimo seis meses. As variáveis estudadas foram: alimentação, sono, linguagem, aquisição de atividades motoras, atividades da vida diária, socialização e reações emocionais. Resultados: das 14 crianças estudadas, $40 \%$ tinham diagnóstico de encefalopatia crônica não progressiva por anóxia perinatal, $14 \%$ tiveram melhora relacionada à alimentação, $23 \%$ ao sono, $43 \%$ à linguagem, $60 \%$ à AVD, $43 \%$ à socialização e $35 \%$ à atenção. Conclusão: A equoterapia é um programa terapêutico muito efetivo na realização de crianças com necessidades humanas básicas afetadas, podendo-se observar melhoras significativas.

\section{AVALIAÇÃO DO CONTEÚdO DE LIVROS ESCOLARES: VACINAS}

\author{
Succi CM, Succi RCM, Wickbold D
}

daniwick@yahoo.com.br

Introdução: Os livros escolares e os professores do ensino fundamental têm papel importante na divulgação dos conceitos de educação em saúde. Objetivo: Assim, o objetivo deste trabalho foi avaliar o conteúdo desses livros sobre conceitos que trazem sobre vacinação. Métodos: Foram analisados 50 livros escolares da área de Ciências e Biologia, onde os autores verificaram o seu conteúdo na área de vacinação. Resultados: Apenas 3 livros da $8^{a}$ série não continham informações sobre saúde, mas 17 livros $(34 \%)$ não continham qualquer informação sobre vacinação. Dos 33 livros com informações sobre vacinas, 19 (57,5\%) continham informações incorretas, 29/33 (87,8\%) associavam vacina com prevenção de doenças, $10 / 33(30,3 \%)$ citavam o calendário básico de vacinação e apenas $7 / 33(21,2 \%)$ informavam sobre a vacinação de outras faixas etárias que não a pediátrica. As vacinas mais citadas foram: vacina contra a poliomielite (26 citações), contra a tuberculose (23), contra o sarampo (21) e DPT (19). Vacinas contra dengue, febre tifóide, cólera e varíola (7 citações) foram citadas mais vezes que as vacinas contra hepatite A (1), gripe (2), varicela (2) e raiva (3). Alguns dos erros encontrados foram, além da citação de vacinas inexistentes (dengue) ou em desuso (varíola), a associação de vacinas com dor e desconforto, o0 conceito de que vacina é um remédio e a indicação de vacinação apenas para crianças. Conclusão: Os livros escolares perdem a oportunidade de introduzir conceitos corretos e adequados sobre imunizações, na época em que as crianças estão ávidas por novos conhecimentos e podem transmití-los para seus familiares. 